\title{
An Analysis of Different Parameters of Architectural Buildings
}

\author{
Neetu Bargoti ${ }^{1}$, A. K. Srivastava ${ }^{2}$ \\ ${ }^{I}$ Research Scholar, Dr.C.V.Raman University Kota, Bilaspur \\ ${ }^{2}$ Prof. \& Head, Dr.C.V.Raman University Kota, Bilaspur
}

\begin{abstract}
Today much importance is given to the acoustical buildings. It plays an important role in creating an acoustically pleasing environment. In this paper an attempt has been given to analysis of different parameters of architectural buildings. Before 1900 A.D. there was no basis of the design of rooms and balls for huge gatherings because the causes of bad hearing were not known to them. In ancient and medieval periods the designs of buildings like Bhuvneshwar temple, Taj Mahal and St. Paul's Cathedral in London etc. in which sound produced kept on reverberating for a pretty long period, were looked upon with an eye of awe, reverence and a piece of admiration and appreciation. On July 30, 2013 devotes caught in a stampede near Baidyanath Dham, on July 15, 1996 Pilgrims died at the Mahakaleshwar Temple Ujjain, \& incident of Uphar Cinema Hall;; June 13,1997 it was totally ignorance of acoustics. This paper describes how the materials like fibre type, fibre size, material thickness, density, airflow resistance and porosity can affect the architectural buildings. At last all the relevant parameters of architectural buildings have been discussed comprehensively.
\end{abstract}

Key words: acoustics, architectural, reverberation, porosity, air flow resistance.

\section{Introduction}

Architectural buildings are related with acoustics. Acoustics is the interdisciplinary science that deals with the study of all mechanical waves in gases, liquids and solids including vibration, sound, ultrasound and infrasound. The earliest records of the study of the fundamental and the first 6 overtones of a vibrating string are attributed to the philosopher Pythagoras in the $6^{\text {th }}$ century BC Aristotle (384-322Bc) understood that sound consisted of contractions and expansions of the air "falling upon and striking the air which is next to it ......" a very good expression of the nature of wave motion. In about $20 \mathrm{BC}$, the Raman architect and engineer Vitruvius wrote a treatise on the acoustic properties discussion of interference, echoes , and reverberation the beginning of architectural acoustics. Galileo Galiei (1564-1642) but also Marin Mersenne (1588-1648), independently discovered the complete laws of vibrating strings. Meanwhile Newton (1642-1727) derived the relationship for wave velocity in solids, a cornerstone of physical acoustics. The eighteenth century saw major advances in acoustics as mathematicians applied the new techniques of calculus to elaborate theories of sound wave propagation. In the nineteenth century the major figures of mathematical acoustics were Helmholtz who consolidated the field of acoustics and Lord Rayleigh in England, who combined the previous knowledge with his own copious contributions to the field in his monumental work "The Theory of sound". The twentieth century saw a burgeoning of technological applications of the large body of scientific knowledge that was by then in place. The first such application was Sabine's ground breaking work in architectural buildings and many others followed. Underwater acoustics was used for detecting submarines in the First World War, sound recording and the telephone played important roles in a global transformation of society. Sound measurement and analysis reached new levels of accuracy and sophistication through the use of electronics and computing. The ultrasonic frequency range enabled wholly new kinds of application in medicinal and industry.

\section{Theoretical Consideration}

There are many parameters related with architectural buildings. Some main points have been pointed out below:-

- The modal theory

- The geometric theory

- The theory of Sabine \& reverberation

- Acoustic intensity and decibel

- Boundary conditions

- $\quad$ Noise effect

- Loudness

- Focusing

- Echelon effect

- Extraneous noise

- Resonance 
- $\quad$ Temperature

- Absorbing material

- Pitch

\subsubsection{The model theory:-}

This theory comes from the homogeneous Helmholtz equation,

$\nabla^{2} \widehat{\phi}+\mathrm{K}^{2} \widehat{\phi}=0$. Considering a simple geometry of a parallelepiped $\left(\mathrm{L}_{1}, \mathrm{~L}_{2}, \mathrm{~L}_{3}\right)$, the solution of this problem is with separated variables :-

$\mathrm{P}(\mathrm{x}, \mathrm{y}, \mathrm{z})=\mathrm{X}(\mathrm{x}) \mathrm{Y}(\mathrm{y}) \mathrm{Z}(\mathrm{z})$

Hence each function $\mathrm{X}, \mathrm{Y}$ and $\mathrm{Z}$ has this form:-

$\mathrm{X}(\mathrm{x})=\mathrm{Ae}^{-\mathrm{ikx}}+\mathrm{Be}^{\mathrm{ikx}}$

with the boundary condition $\frac{\partial p}{\partial x}=0$, for

$\mathrm{x}=0$ and $\mathrm{x}=\mathrm{L}_{1}$ (idem in the directions),

the expression of pressure is :

$\mathrm{P}(\mathrm{x}, \mathrm{y}, \mathrm{z})=\mathrm{C} \cos \left(\frac{m \pi x}{L_{1}}\right) \cos \left(\frac{n \pi y}{L_{2}}\right) \cos \left(\frac{p \pi z}{L_{3}}\right)$

$\mathrm{K}^{2}=\left(\frac{m \pi}{L_{1}}\right)^{2}+\left(\frac{n \pi}{L_{2}}\right)^{2}+\left(\frac{p \pi}{L_{3}}\right)^{2}$

Where, $\mathrm{m} \mathrm{n}, \mathrm{p}$ are whole numbers. It is three directional stationary wave acoustic modes appear with their model frequencies and their modal forms. With a non-homogeneous problem, a problem with an acoustic source $Q$ in $r_{0}$, the final pressure in $r$ is the sum of the contribution of all the modes described above. The modal density $\frac{d N}{d f}$ is the number of modal frequencies contained in a range of $1 \mathrm{~Hz}$. It depends on the frequency $\mathrm{f}$, the volume of the room $\mathrm{v}$ and the speed of sound $\mathrm{c}_{0}$ :

$\frac{d \mathrm{~N}}{d f} \cong \frac{4 \pi v}{c_{0}^{2}} \mathrm{f}^{2}$

The modal density depends on the square frequency, so it increases rapidly with the frequency. At a certain level of frequency, the modes are not distinguished and the model theory is no longer relevant.

\subsubsection{The geometry theory:}

For rooms of high volume or with a complex geometry, the theory of acoustical geometry is critical and can be applied. The waves are modelised with rays carrying acoustical energy. This energy decrease with the reflection of the rays on the walls of the rooms. The reason of this phenomenon is the absorption of the walls. The problem is this theory needs a very high power of calculation and that is why the theory of Sabine is often chosen because it is easier.

\subsubsection{The theory of Sabine \& reverberation:}

This theory uses the hypothesis of the diffuse field; the acoustical field is homogeneous and isotropic. In order to obtain this field the room has to be enough reverberating and the frequencies have to be high enough to avoid the effects of predominating modes. The variation of the acoustical energy $\mathrm{E}$ in the room can be written as :$\frac{d E}{d t}=\mathrm{W}_{\mathrm{s}}-\mathrm{W}_{\mathrm{abs}}$

where $\mathrm{W}_{\mathrm{s}}$ and $\mathrm{W}_{\mathrm{abs}}$ are respectively the power generated by the acoustical source and the power absorbed by the walls. The power absorbed is related to the voluminal energy in the room e : Ws $=\frac{\theta 60}{4} \mathrm{a}$

Where $\mathrm{a}$ is the equivalent absorption area defined by the sum of the product of the absorption coefficient and the area of each material in the room:

$\mathrm{a}=\sum \propto_{\mathrm{i}} s_{\mathrm{i}}$

The final equation is,

$\mathrm{V} \frac{d e}{d t}=\mathrm{Ws}-\frac{e c 0}{4} \mathrm{a}$

The level of stationary energy is :-

$\mathrm{e}_{\text {sat }}=4 \frac{W_{s}}{a c_{0}}$

The sound waves received by the listener are : (i) direct waves and (ii) reflected waves due to multiple reflections at the various surfaces. The quality of the note received by the listener will be the combined effect of 
these two sets of waves. There is also a time gap between the direct wave received by the listener and the waves received by successive reflection. Due to this, the sound persists for some time ever after the source has stopped. This persistence of sound is termed as reverberation. The time gap between the initial direct note and the reflected note up to the minimum audibility level is called reverberation time. The reverberation time depends on the size of the room or the auditorium the nature of the reflecting material on the wall and the ceiling and the area of the reflecting surfaces. In a good auditorium it is necessary to keep the reverberation time negligibly small.

With this theory described, the reverberation time can be defined. It is the time for the level of energy to decrease of $60 \mathrm{~dB}$. It depends on the volume of the room $\mathrm{v}$ and the equivalent absorption area.

$$
\mathrm{T}_{60}=\frac{0.16 \mathrm{v}}{a}
$$

This reverberation time is the fundamental parameter in room acoustics and depends through the equivalent absorption area and the absorption acoustic coefficients on the frequency.

\subsubsection{Intensity and decibel:-}

$\mathrm{i}(\mathrm{t})=\mathrm{Pv}$

The acoustic intensity represents the acoustic energy flux associated with the wave propagation:

we can then define the average intensity :

$\mathrm{I}=\langle\mathrm{i}\rangle$

However, acoustic intensity does not give a good idea of the sound level, since the sensitivity of our ears is logarithmic. Therefore we define decibels, either using acoustic over pressure or acoustic average intensity :-

$p^{d B}=20 \log \left(\frac{p}{\text { Pref }}\right)$
$L_{l}=10 \log \left(\frac{I}{\text { Iref }}\right)$

where

$\mathrm{P}_{\text {ref }}=2.10^{-5} \mathrm{~Pa}$ for air

or $\mathrm{p}_{\mathrm{ref}}=10^{-6} \mathrm{~Pa}$ for any other media

and $\mathrm{I}_{\mathrm{ref}}=10^{-12} \mathrm{~W} / \mathrm{m}^{2}$

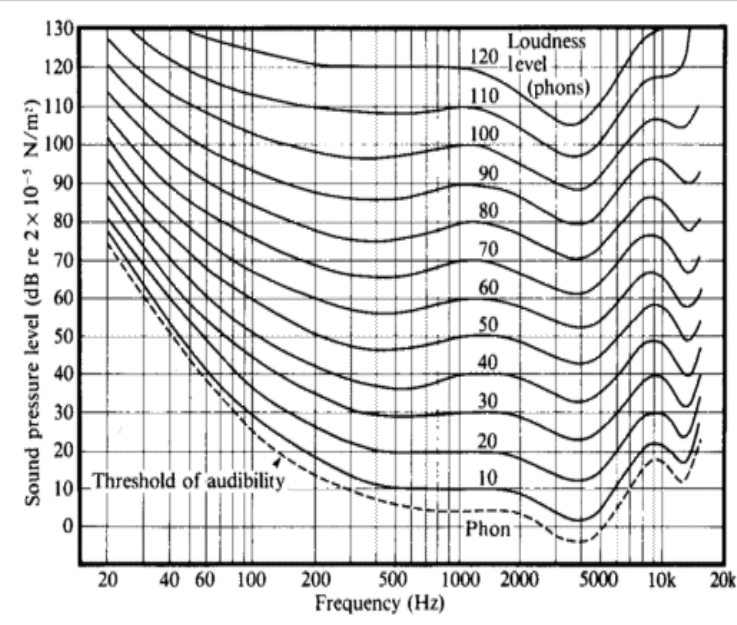



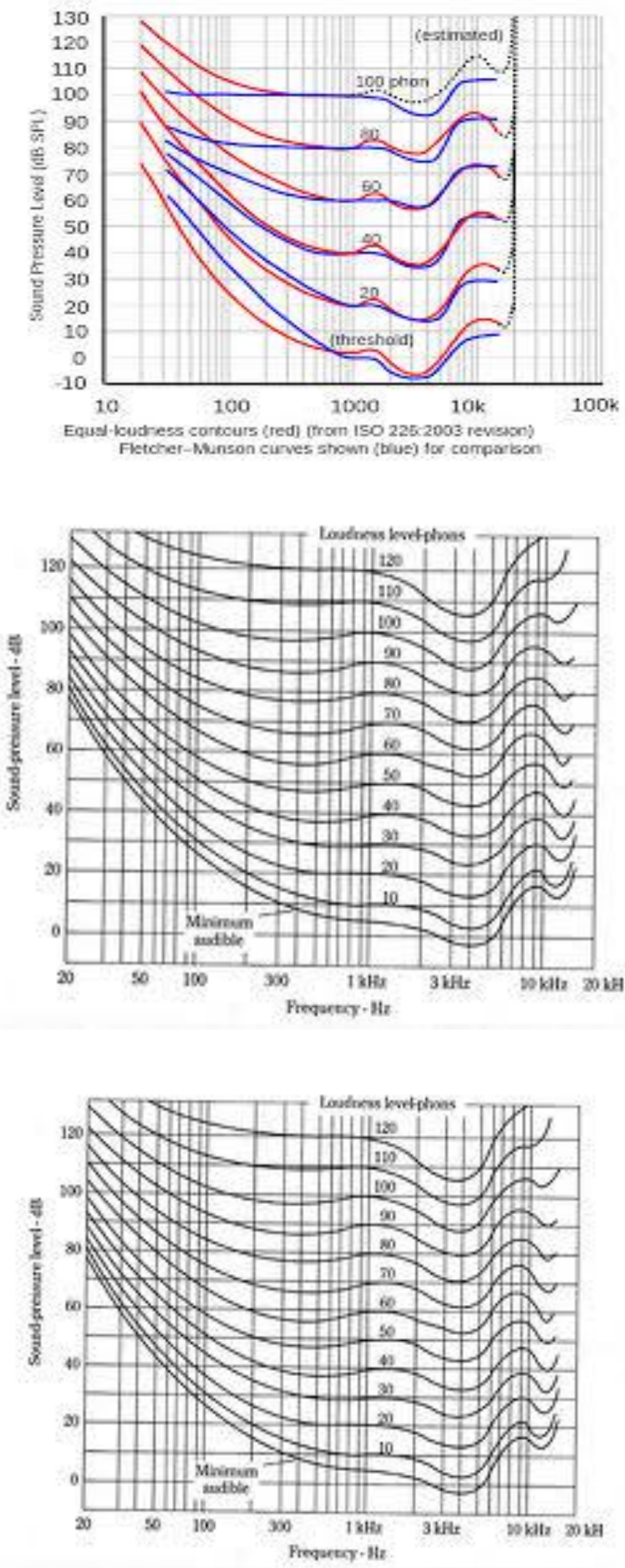

\subsubsection{Boundary condition:-}

Concerning the boundary conditions which are used for solving the wave equation, we can distinguish two situations. If the medium is not absorptive, the boundary conditions are established using the usual equations for mechanics. But in the situation of an absorptive material, it is simples to use the concept of acoustic to use the concept impedance. In that case we get explicit boundary conditions either on stresses or on velocities at the interface. These conditions depend on whether the media are solids, in viscid or viscous fluids. Here we use the acoustic impedance as the boundary conditions. This impedance, which is often given by experimental measurement, depends on the material, the fluid and the frequency of the sound wave.

\subsubsection{Noise effect:-}

Noise comprises all sounds, which due to their loudness and structure, are considered as harmful for human beings and environment. It depends on the condition, preferences and mood a person whether sounds are perceived as noise or not. Noise effect plays vital role. 


\subsubsection{Loudness:-}

It is the characteristic of sound by virtue of which we distinguish two sounds of the same frequency. It indicates the slowness and highness of sound. Loudness depends upon the following points as well as:

- density of air

- Velocity and direction of wind

- Source

- Sensitivity of the ear.

The human ear is remarkable both in terms of its absolute sensitivity and in terms of the range of intensities to which it can respond.

\subsubsection{Focusing:} focusing.

The presence of cylindrical or spherical surfaces an the walls or the cussing gives rise to undesirable

\subsubsection{Echelon effect:}

If there is regular structure similar to a flight of stairs or a set of railings in the hall, the sound produced in front of such a structure may produce musical note due to regular successive echoes of sound reaching the observer. Such on effect is celled echelon effect.

\subsubsection{Extraneous noise :}

The extraneous noise may be due to (a) sound received from outside the room (b) the sound produced by the fans etc. Clearly it is unwanted sound.

\subsubsection{Resonance:}

The acoustics of a building may also be affected by resonance. If there is resonance for any audio frequency note, the intensity of the note will be entirely different from the intensity desired.

\subsubsection{Tolerance of temperature:-}

Tolerance of temperature in material should be high. In another words specific type of materials which have high temperature tolerance should be used. Some unwanted mishappening can be avoided by their technique.

\subsubsection{Absorbing material:-}

Materials that reduce the acoustic energy of a sound wave as the wave passes through it by the phenomenon of absorption are called sound absorptive materials. Absorptive materials are generally resistive in nature, either fibrous, porous or in rather special cases reactive resonators. Lewis H. Bell, 1994 classic example of resistive material are nonwovens, fibrous glass, mineral wools, felt and foams porous materials used for noise control are generally categorized as fibrous medium or porous foam.

Fibrous media usually consists of glass, rock wool or polyester fibres and have high acoustic absorption. Some time fire resistant fibres are also used in making acoustical product, (Claudio, 1998). Acoustical material plays a number of roles that are important in acoustical building such as the control of room acoustic, studio acoustics etc. Sound absorptive materials may also be used to control the response of artistic performance spaces to steady and transient sound sources, thereby affecting the character of the aural environment, the intelligibility of unreinforced speech (frank July 2001). There are several factors influences sound absorption i.e. fibre size, air flow resistance, porosity, tortuosity, thickness, density, compression, surface impedance, placement/position of sound absorptive, performance of absorbing material etc. Marble, carpet, acoustic plaster, acoustic felt, fibre board, heavy curtains, fibre glass, perforated cellulose fibre tiles are the absorbing material.

\subsubsection{Pitch:}

It is also called shrillness of sound . It is that characteristic of sound by which an acute or shrill note can be distinguished from a grave or a flat one, If the frequency of vibration of a sonorous body is high, the pitch as sensed by a listener, will be high; if the frequency is low, the pitch will be low. All musical notes have a definite pitch.

\section{Conclusion}

An understanding of several area of material science and acoustics is necessary to successfully develop materials for different parameters of architectural buildings. Really the influence of various parameters of 
acoustic materials is presented as well as summarized. Some of the important conclusions of this research are as follow:

Fiber surface area and fibre size have strong influence on sound absorption properties, higher surface area and lower fibre size increases sound absorption. Films such as PVC attachment increase sound absorption at low and mid frequencies at the expense of higher frequencies. Less dense and more open structure absorbs sound of low frequencies $\left(500 \mathrm{H}_{\mathrm{Z}}\right)$, denser structure performs better for frequencies above than $2000 \mathrm{H}_{\mathrm{z}}$

\section{References:}

[1]. Claudio Braccesi and Anderea Bracciali "Least Squares Estimation of Main Properties of Sound Absorbing Materials Through Acoustical Applied Acoustic Measurements "54 (i) : 59-70, 1998.

[2]. Francisco Simon and Jaime Pfretzschner "Guidelines for the Acoustic Design of Absorptive Devices, Noise and Vibration World Wide " 2004.

[3]. Frank, Fahy; Foundation of Engineering Acoustics " San Diego, Calif ; London: $\quad$ Academic Press. 2001.

[4]. Knapen E.R etal, "Sound Absorption by Polymer, Modified Porous Cement Mortars" $6^{\text {th }}$ International Conference on Materials Science and Restoration MSR-vi Aedificatio Publishers pp:347-358, 2003.

[5]. Koizumi T, N. Tsugeuchi and A Adachi "The Development of Sound Absorbing Materials using Natural Bamboo Fibres, High Performance" WIT Press , 2002.

[6]. Lewis H., Bell, Industrial Notise Control, Fundamentals and applications, 2nd edition, New york M. Dekker, 1994.

[7]. Mingzhang Ren and firm Jacobsen, "A Method of Measuring The Dynamic flow Resitance and Reactance of Porous Materials" , Applied Acoustics 39(4); 265-265, 1993.

[8]. Durga Prasad singh “ Waves \& Acoustics, Edition : 1998, Student Friends Educational Publishers, Patna .

[9]. N. Subrahmanyam, Brij Lal, “A Text Book of Sound” Second Revised Edition 1995 Vikas Publishing House Pvt. Ltd. New Delhi Reprint 2002.

[10]. A.K. Datta, N.R. Ganguli etal, “ Speech and Music-A Review on Quantitative Studies” Jour . Sangeet Research Academy 4, pp.77$91,1983$.

[11]. http://www. engineeringtoolbov.com/acoustics-sound. absorption -d_68 html.

[12]. http://en.wikipedia.org/wiki/Uphar_cinema_fire. 